\title{
Soluble a-proliferation-inducing ligand (sAPRIL), a novel serum biomarker predicting the recurrence and metastasis of pancreatic adenocarcinoma after surgery
}

\author{
LEI HAN $^{1 *}$, WEI ZHANG ${ }^{1 *}$, FULIN SONG ${ }^{2}$, YANG GUO ${ }^{1}$, KEJIAN GUO $^{3}$ and WENPING ZHOU ${ }^{1}$ \\ Departments of ${ }^{1}$ Hepatobiliary and Pancreas Surgery and ${ }^{2}$ Pathology, The General Hospital of Shenyang Military Region, \\ Shenyang, Liaoning 110016; ${ }^{3}$ Department of General Surgery, College of Clinical Medical Sciences, \\ China Medical University, Shenyang, Liaoning 110004, P.R. China
}

Received November 30, 2013; Accepted May 19, 2014

DOI: $10.3892 / \mathrm{mmr} .2014 .2443$

\begin{abstract}
Pancreatic adenocarcinoma (PA) is a leading cause of adult cancer mortality, and surgery is still the best available treatment strategy. However, PA can recur at any time and has limited prognosis. It is therefore necessary to explore novel serum biomarkers of PA to allow the early diagnosis of PA. Soluble a-proliferation-inducing ligand (sAPRIL), a promising inducer of the epithelial-mesenchymal transition (EMT), is often found overexpressed in a variety of autoimmune diseases. To determine whether serum sAPRIL can constitute a PA biomarker, the protein level of sAPRIL was examined by immunohistochemistry and western blot, and the mRNA level was quantified by RT-qPCR. The PA cell line PanC-1 was transfected with vectors bearing the sAPRIL gene and sAPRIL short hairpin RNA (shRNA) oligos. Increased expression of serum SAPRIL was observed in patients with PA recurrence or metastasis after five-year surgery compared to subjects without PA recurrence or metastasis. The growth rate of PanC-1 cells transfected with the $S A P R I L$ expression vector was increased by $23 \%$ ( $\mathrm{P}<0.01$, vs. control group), and was reduced by $17 \%(\mathrm{P}<0.01$, vs. control group) in the sAPRIL shRNA-silenced cell line. Thus, sAPRIL is highly expressed in PA, and serum levels of sAPRIL can serve as a useful indicator for the recurrence or metastasis of PA after surgery. Additional validation studies on the use of serum SAPRIL as a diagnostic marker in PA are however needed.
\end{abstract}

Correspondence to: Dr Wenping Zhou, Department of Hepatobiliary and Pancreas Surgery, The General Hospital of Shenyang Military Region, No. 83 Wenhua Road, Shenhe, Shenyang, Liaoning 110016, P.R. China

E-mail: wenpingzhousy@163.com

*Contributed equally

Key words: pancreatic adenocarcinomas, serum biomarkers, soluble a-proliferation-inducing ligand, recurrence, metastasis, surgery

\section{Introduction}

Pancreatic adenocarcinoma (PA) is one of the leading causes of cancer-related mortality and has considerable economic and social impact (1). The lethality of the disease is largely caused by difficulties in early detection, and most PA cases remain incurable by currently available treatment strategies. A high proportion of PA patients show locally advanced or metastatic tumor growth. Considerable efforts have been made during the past decade to identify improved systemic treatment options. However, clinical trials have not identified a survival advantage for the majority of tested therapies (2).

Pathological diagnosis is a key factor for the effective and timely treatment of PA. Histological diagnosis of cystic pancreatic lesions (CPLs) is often difficult, because of the low sensitivity of fine needle aspiration biopsy and brush cytology. Direct intracystic biopsy and pancreatic cystoscopy are still the standard practice (3). However, such biopsies are invasive and therefore difficult to obtain. By consequence, certain candidate biomarkers for PA are emerging. For instance, the intracellular anti-apoptotic protein BAG3 was found to be expressed in pancreatic ductal adenocarcinoma (PDAC) samples, and not in the surrounding non-neoplastic tissue or in healthy pancreatic tissue. Furthermore, in a cohort of patients who underwent R0 resection, the level of BAG3 inversely correlated to patient survival. BAG3 protein was also present in the patients' sera, and was thus proposed as a useful biomarker for PDAC (4). In another example, Kosanam et al performed Orbitrap(R) mass spectrometry proteomic analysis of four PDAC tissues and adjacent benign tissues. A significant elevation of LAMC2 was observed in the serum of patients with PA (5). Although several biomarkers for PA have been proposed (5-13), no single marker has emerged as the test of choice. Further research and better understanding of the biology of PA, improved diagnostic techniques, and standardized interpretation are essential steps to develop reliable biomarkers.

Compared to other types of biomarkers, an advantage of serum biomarkers is that they can be measured by less invasive methods. The soluble a proliferation-inducing ligand (sAPRIL) protein is a member of the tumor necrosis factor (TNF) family, comprising proteins that regulate B-cell maturation, 
survival, and function (14). Epithelial-mesenchymal transition (EMT) was shown to be upregulated by tumour necrosis factor- $\alpha(\mathrm{TNF}-\alpha)$ and to relate to the recurrence of certain types of cancer $(15,16)$. Thus, sAPRIL, which is often found overexpressed in a variety of autoimmune diseases (17) may be associated with MET. sAPRIL has been reported to stimulate tumor cell growth (14), but the function of SAPRIL in PA has not been reported to date. The serum levels of APRIL have been widely studied in numerous patients in order to explore its clinical significance (18-23). sAPRIL is the active form of APRIL and serum SAPRIL was previously detected in colorectal cancer (24). Here, we aimed to explore the potential of using the serum sAPRIL level as a biomarker for the prediction of PA occurrence and metastasis, which would contribute to the early diagnosis of PA following surgery.

\section{Materials and methods}

Patient samples and cell lines. All protocols were approved by the ethics committee of The General Hospital of Shenyang Military Region (Shenyang, China). Written informed consent was obtained from all patients. From March 2005 to March 2008, a total of 448 patients were diagnosed with PA, and 400 of these subsequently underwent a standardized Kausch-Whipple resection. Blood samples were obtained preoperatively from five-year surviving patients and were processed according to a standardized protocol. Serum samples were immediately frozen in aliquots at $-80^{\circ} \mathrm{C}$, and the excised pancreatic tissue samples were placed in liquid nitrogen and stored at $-80^{\circ} \mathrm{C}$ until use. PA diagnosis was performed histologically in all patients. None of the patients received any additional therapy. The final stages of pancreatic cancer were examined histopathologically according to the general rules of the TNM classification system of malignant tumors, defined by the International Union Against Cancer (UICC) (25). The PA cell line PanC-1 (American Type Culture Collection, Manassas, VA, USA) was cultured in serum-free RPMI-1640 medium (Product no. R6504, Sigma-Aldrich, St. Louis, MO, USA) in a humidified atmosphere containing $5 \%$ $\mathrm{CO}_{2}$ at $37^{\circ} \mathrm{C}$.

Immunohistochemistry. Immunohistochemistry was performed according to a previously reported method (26). Briefly, 4-mm-thick serial sections were performed on paraffin-embedded pancreatic tissues and were deparaffinized. These slices were placed in $10 \mathrm{mM}$ citric acid buffer ( $\mathrm{pH}$ 6.0) with $0.2 \%$ Tween-20 and boiled in a microwave oven $(6 \mathrm{~min})$ to retrieve the antigen. The slides were then rinsed and blocked in a $10 \% \mathrm{H}_{2} \mathrm{O}_{2}$ solution with methanol for $10 \mathrm{~min}$. Subsequently, the slices were incubated with mouse anti-human sAPRIL monoclonal antibody (1:500 dilution; Product no. LS-C126875, LifeSpan Biosciences, Inc., Seattle, WA, USA) overnight at $4^{\circ} \mathrm{C}$. They were then rinsed in phosphate-buffered saline, and incubated for $1 \mathrm{~h}$ with secondary antibody labeled with streptavidin-biotin-peroxidase (DAKO LSAB $^{\text {тм }} 2$ kit; DakoCytomation, Glostrup, Denmark). The bound complex was visualized using as a chromogen liquid diaminobenzidine, and counterstained with hematoxylin and eosin stain kit (Shanghai Haoran Bio Technologies Co., Ltd., Shanghai, China).
sAPRIL expression construct. Genomic DNA from PA tissues was extracted using the Genomic DNA Extraction kit (Takara, Dalian, China), following the manufacturer's instructions. The $s A P R I L$ gene was amplified from genomic DNA using the following primers: sense, 5'-GTG AGC TAG CAT GCC AGC CTC ATC TCC AGG CCA CAT G-3', and antisense, 5'-CTG AGA ATT CTT ATA GTT TCA CAA ACC CCA GGA ATG-3'. The sAPRIL gene was amplified on an Applied Biosystems ${ }^{\circledR}$ Thermal Cycler 2720 (Invitrogen Life Technologies, Carlsbad, CA, USA) using the following parameters: One cycle of an initial denaturation step at $95^{\circ} \mathrm{C}$ for $4 \mathrm{~min}$; followed by 25 cycles consisting of $30 \mathrm{sec}$ at $94^{\circ} \mathrm{C}, 30 \mathrm{sec}$ at $55^{\circ} \mathrm{C}$ and $1 \mathrm{~min}$ at $72^{\circ} \mathrm{C}$; a final extension step of $10 \mathrm{~min}$ at $72^{\circ} \mathrm{C}$, which generated a 750-bp product. The PCR product was cloned into the NheI-EcoRI sites of the pcDNA3.1 vector (TOPO TA $^{\circledR}$ Expression Kit, Applied Biosystems China Ltd., Beijing, China), giving the plasmid named as pcDNA3.1-sAPRIL. This plasmid was then introduced and expressed in E. coli cells, isolated using the MiniBEST Plasmid Purification kit ver.4.0 (Takara Biotechnology Co., Ltd., Dalian, China) and the cloned sequence of the $S A P R I L$ gene was verified by sequencing.

Construct for sAPRIL gene silencing. The pTZU6+1 expression vector was a gift from the Shandong Provincial Institute of Parasitic Diseases (Jining, Shandong, China). The $s A P R I L$ coding and reverse complementary sequences were amplified from genomic DNA with the following primers: sense, 5'-TCG ACC TGG GTG AGT ACT GCT CTC CTT GGG GAG AGC AGT ACT CAC CCA GTT TTT T-3', and antisense, 5'-CTA GAA AAA ACT GGG TGA GTA CTG CTC TCC CCA AGG AGA GCA GTA CTC ACC CAG-3'. PCR was performed as described above. SalI and $\mathrm{XbaI}$ restriction sites were incorporated on either end of the oligos for cloning into the pTZU 6+1 vector, providing the pTZU6+1-shRNA-sAPRIL plasmid.

Transfection of the PanC-1 PA cell line. PanC-1 cells $\left(2 \times 10^{5} /\right.$ plate) were transfected with the pcDNA3.1-sAPRIL and the pTZU6+1-sAPRIL vectors. Non-transfected cells served as the control group. Transfection was performed in $50 \%$ confluent cells using $9 \mu 1$ of Lipofectamine $2000^{\mathrm{TM}}$ (Applied Biosystems Trading Co. Ltd., Shanghai, China). Following a 40-h transfection, neomycin-resistant clones were selected following addition of $500 \mu \mathrm{g} / \mathrm{ml} \mathrm{G} 418$ (product no. 1453C082; Beijing Jingke Hongda Biotechnology Co., Ltd., Beijing, China) in the medium. Resistant colonies were either pooled or cloned.

Reverse transcription-quantitative PCR (RT-qPCR). Total RNA was extracted from pancreatic tissues with the RNeasy Mini Kit (Qiagen, Shanghai, China) according to the manufacturer's instructions. cDNA was synthesized from $1 \mathrm{mg}$ of total RNA with the T-Primed First-Strand kit for RT-PCR (GE Healthcare Life Sciences, Shanghai, China). The level of SAPRIL mRNA was evaluated by qPCR. The primer sequences for gene amplification were the following: sAPRIL forward, 5'-AGT CCT GCA TCT TGT TCC AG-3', and reverse, 5'-AGA GAA ACT CTA TTC CGA TG-3'; GAPDH forward, 5'-CCC TTC ATT GAC CTC AAC TAC-3,' and reverse, 5'-CCA CCT TCT TGA TGT CAT CAT-3'. GAPDH was used for normalization. The qPCR was performed applying an SYBR Green I 
Table I. Prognostic factors of the five-year survivors of pancreatic adenocarcinoma (PA) following surgery.

\begin{tabular}{|c|c|c|c|c|}
\hline \multirow[b]{2}{*}{ Prognostic factors } & \multirow[b]{2}{*}{ Number } & \multicolumn{2}{|r|}{ sAPRIL level } & \multirow[b]{2}{*}{ P-value } \\
\hline & & Light density & Protein concentration $(\mathrm{ng} / \mathrm{ml})$ & \\
\hline \multicolumn{5}{|l|}{ Age } \\
\hline$<60$ & 45 & $0.0024 \pm 0.0022$ & $13.1 \pm 10.2$ & $0.64 / 0.46$ \\
\hline$\geq 60$ & 35 & $0.0026 \pm 0.0033$ & $14.2 \pm 11.9$ & \\
\hline \multicolumn{5}{|l|}{ Gender } \\
\hline Male & 50 & $0.0027 \pm 0.0032$ & $13.8 \pm 5.0 .$. & $0.88 / 0.57$ \\
\hline Female & 30 & $0.0023 \pm 0.0029$ & $14.0 \pm 4.5 .$. & \\
\hline \multicolumn{5}{|l|}{ Tumor size } \\
\hline$<3 \mathrm{~cm}$ & 47 & $0.0021 \pm 0.0013$ & $6.1 \pm 1.8$ & $0.57 / 0.82$ \\
\hline$\geq 3 \mathrm{~cm}$ & 33 & $0.0039 \pm 0.0033$ & $28.4 \pm 6.7 .$. & \\
\hline \multicolumn{5}{|l|}{ TNM stage } \\
\hline I + IIA & 41 & $0.0005 \pm 0.0002$ & $5.7 \pm 1.5$ & $0.001 / 0.006$ \\
\hline $\mathrm{IIB}+\mathrm{III}+\mathrm{IV}$ & 39 & $0.0043 \pm 0.0034$ & $30 \pm 7.1$ & \\
\hline \multicolumn{5}{|c|}{ Lymphoid knot transfer } \\
\hline Yes & 57 & $0.0045 \pm 0.0032$ & $31 \pm 8.3$ & $0.001 / 0.006$ \\
\hline No & 23 & $0.0005 \pm 0.0002$ & $5.9 \pm 1.7$ & \\
\hline \multicolumn{5}{|c|}{ Number of lymphoid knot transfer } \\
\hline$<3$ & 31 & $0.0036 \pm 0.0025$ & $8 \pm 7.7$ & $0.005 / 0.007$ \\
\hline$\geq 3$ & 26 & $0.0112 \pm 0.0060$ & $25 \pm 10.4$ & \\
\hline \multicolumn{5}{|l|}{ Type of tissues } \\
\hline Tumor & 80 & $0.0028 \pm 0.0021$ & $14.8 \pm 12.1$ & $0.001 / 0.004$ \\
\hline Healthy & 80 & $0.0009 \pm 0.0010$ & $4.3 \pm 3.2$ & \\
\hline
\end{tabular}

$\mathrm{P}$-values are presented as a ratio. The ratio represents the P-value of light density and the P-value of protein concentraton.

reaction mix on a Real-Time Thermal Cycler (qTOWER 2.2; Analytik Jena AG, Jena, Germany). Data analyses were performed based on the standard curve and $\Delta \Delta \mathrm{CT}$ method.

Estimation of growth rate of PanC-1 cells. A clean hemocytometer and cover slip were prepared. In order to count accurately, the single cells were obtained by pipetting up and down in a tube. Subsequently, $50 \mu 1$ of the cell suspension was placed over the counting chambers and the entire hemocytometer was filled. The cells viewed under a microscope (magnification, x100). Cells were counted on both sides of the chambers. Each area of the grid was $0.0001 \mathrm{ml}$ and all cells were counted within four areas. The growth rate of transfected and non-transfected PanC-1 cells was estimated at each 24-h interval according to the cell concentration.

Western blot analysis. The extracellular proteins were isolated from cell culture supernatants of PanC-1 cells. Firstly, the culture solution was centrifuged at $900 \mathrm{x}$ g for $5 \mathrm{~min}$ to collect the supernatants and cells separately. Secondly, the collected supernatants were further centrifuged at $10,000 \mathrm{x} g$ for $1 \mathrm{~h}$ to remove the remaining debris. The supernatants were filtered through $0.2 \mu \mathrm{m}$ pore filters. Finally, the supernatants were concentrated between $1 \mathrm{ml}$ and $100 \mu \mathrm{l}$ using 2-ml Amicon Ultra centrifugal filtration units (UFC201024, 10,000 NMWL, EMD Millipore Headquarters, Billerica, MA, USA). The intracellular proteins were isolated from cells by subjecting to three freeze-thaw cycles, each consisting of $5 \mathrm{~min}$ of freezing at $-80^{\circ} \mathrm{C}$ and $10 \mathrm{~min}$ of thawing at $37^{\circ} \mathrm{C}$ in PBS buffer. The extracted proteins from frozen tissue samples and cultured cells were subjected to western blot analysis using the mouse anti-human monoclonal antibody targeting sAPRIL (Shanghai Institute of Biochemistry and Cell Biology, CAS, Shanghai, China). The antibody targeting $\beta$-actin was purchased from Abcam (Beijing, China) and used as the loading control. The non-transfected and transfected cell lines and pancreatic tissues were homogenized in RIPA buffer $(150 \mathrm{mM}$ sodium chloride, $1 \% \mathrm{NP}-40,0.5 \%$ sodium deoxycholate, $0.1 \%$ SDS, $50 \mathrm{mM}$ Tris- $\mathrm{HCl}, \mathrm{pH}$ 8.0) supplemented with the serine protease inhibitor phenylmethanesulfonyl fluoride (Yaxin Biotechnology Co., Ltd., Shanghai, China). Total protein was extracted from the cell lysate using cell lysis buffer $(50 \mathrm{mM}$ HEPES pH 7.0, $150 \mathrm{mM} \mathrm{NaCl}, 1$ mM EDTA, 1 mM EDTA, $1 \mathrm{mM}$ DTT and $0.1 \%$ Triton $\mathrm{X}-100)$. The protein concentration was determined using a BCA assay kit (Thermo Scientific, Rockford, IL, USA) according to the manufacturer's instructions. The proteins were separated by sodium dodecyl sulfate-polyacrylamide gel electrophoresis and transferred onto a polyvinylidene fluoride membrane (EMD Millipore, Billerica, MA, USA). The membranes were blocked with $5 \%$ skim milk in TBST $(10 \mathrm{mM}$ Tris, $\mathrm{pH} 7.5,100 \mathrm{mM} \mathrm{NaCl}$ and $0.1 \%$ Tween-20) and incubated with the anti-sAPRIL 

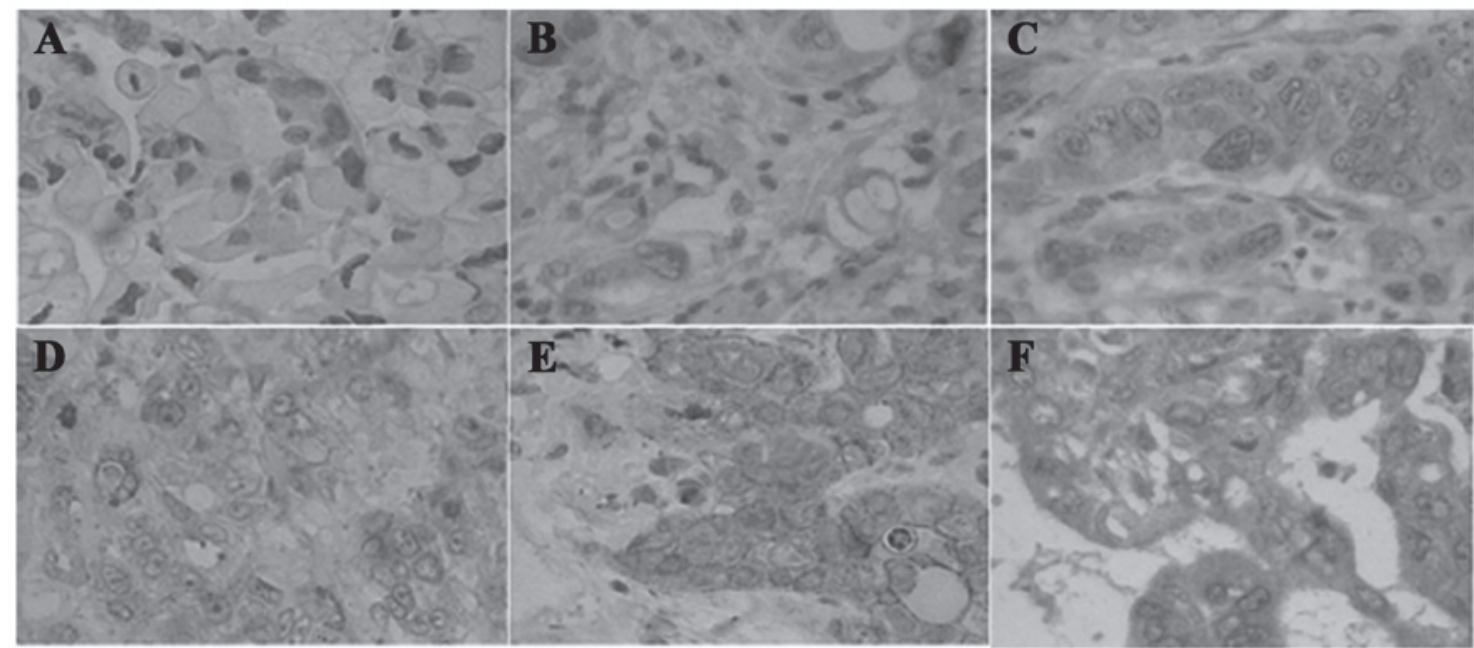

Figure 1. Immunohistochemistrical analysis of sAPRIL in PA tissues at different stages of the disease. (A) Healthy pancreatic tissue; (B) TNM stage I; (C) TNM stage IIA; (D) TNM stage IIB; (E) TNM stage III; (F) TNM stage IV. sAPRIL, soluble a-proliferation-inducing ligand; PA, pancreatic adenocarcinoma.

A
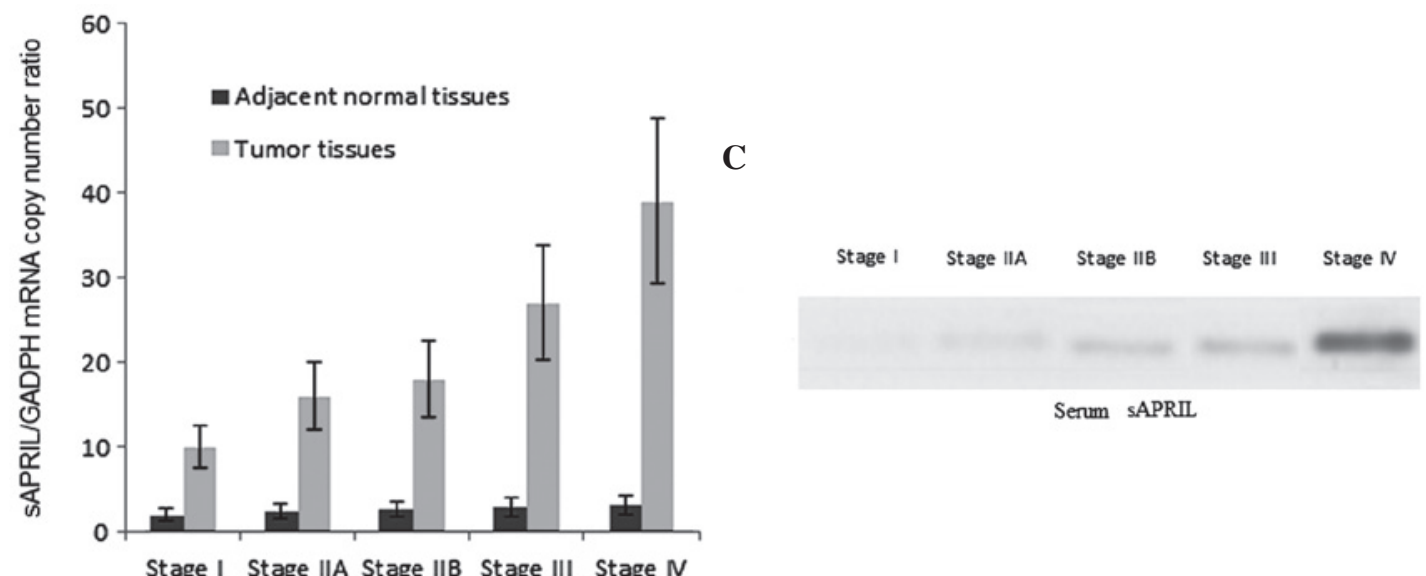

B

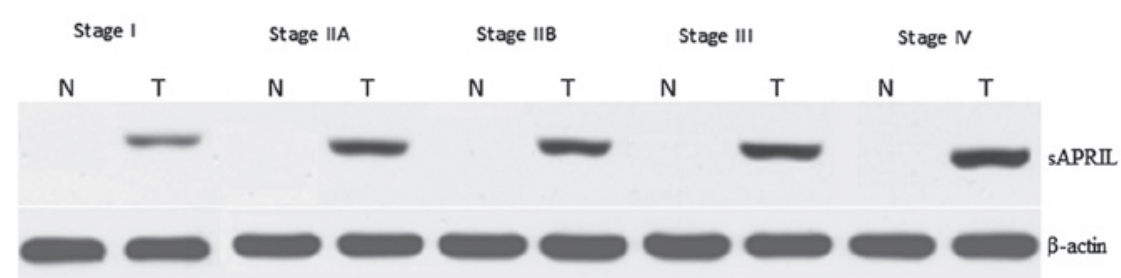

Figure 2. Reverse transcription-quantitative PCR (RT-qPCR) and western blot analysis of sAPRIL in PA and adjacent healthy (normal) pancreatic tissues. (A) RT-qPCR analysis shows higher levels of $s A P R I L$ mRNA in PA tissues than in adjacent healthy pancreatic tissues. Each bar represents the mean \pm SD of three independent experiments. (B) Western blot analysis shows abundant sAPRIL expression in PA tissues (T), but not in adjacent healthy pancreatic tissues (N) in all cases examined. $\beta$-actin was used as the loading control. (C) Western blot analysis shows that the serum sAPRIL level increases with the development of PA. sAPRIL, soluble a-proliferation-inducing ligand; PA, pancreatic adenocarcinoma.

antibody overnight at $4^{\circ} \mathrm{C}$. The membrane was then incubated with horseradish peroxidase (HRP)-conjugated secondary antibody (1:3,000, GE Healthcare, Piscataway, NJ, USA). Immunoreactive bands were visualized using an enchanced chemiluminescence kit (GE Healthcare, Shanghai, China) and were quantified by densitometry with the Image $\mathrm{J}$ software 1.45 (National Institutes of Health, Bethesda, MD, USA).

Statistical analysis. Statistical analyses were carried out using the appropriate tests. The long-term disease outcome and the prognostic sAPRIL factor were analyzed by multivariate and univariate analyses using SPSS software package (SPSS 13.0; SPSS, Inc., Chicago, IL, USA). P<0.05 was considered to indicate a statistically significant difference.

\section{Results}

Features of the five-year PA survivor patients. Among 400 patients with PA, the five-year postoperative mortality was $70 \%$ and the morbidity was $20 \%$. A good outcome, without 
A

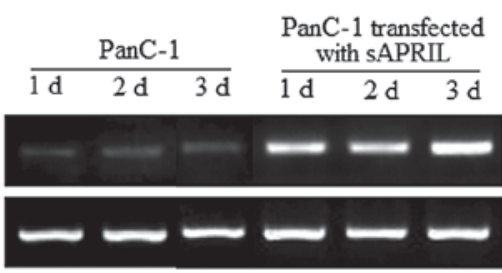

C

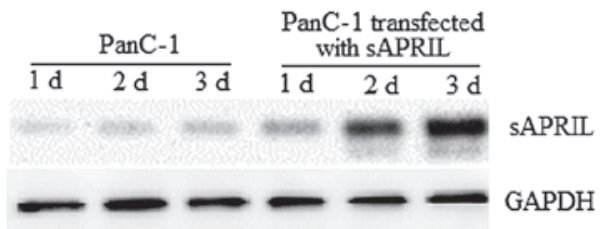

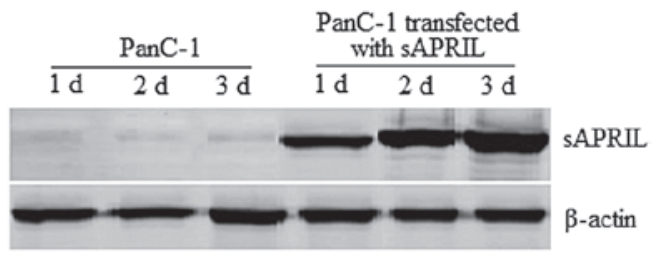

D

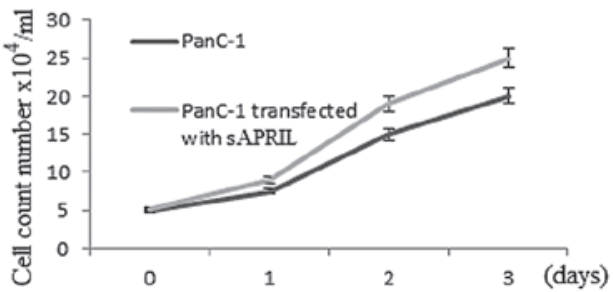

Figure 3. sAPRIL levels in PanC-1 cells transfected with the sAPRIL expression vector, and growth rate of transfected cells. (A) Reverse transcription-quantitative PCR analysis reveals higher levels of the sAPRIL mRNA in transfected compared to non-transfected PanC-1 cells. (B) Western blot showing higher levels of the sAPRIL protein in transfected compared to non-transfected PanC-1 cells. (C) Western blot analysis of the levels of sAPRIL in the serum of non-transfected and transfected PanC-1 cells. (D) Transfection with the SAPRIL expression vector increases the growth rate of transfected cells compared to non-transfected PanC-1 cells. Each bar represents the mean \pm SD of three independent experiments. sAPRIL, soluble a-proliferation-inducing ligand.

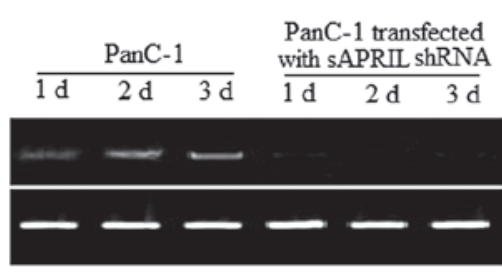

C

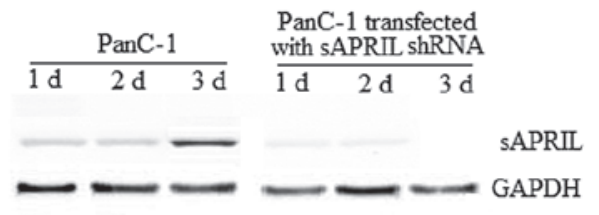

B

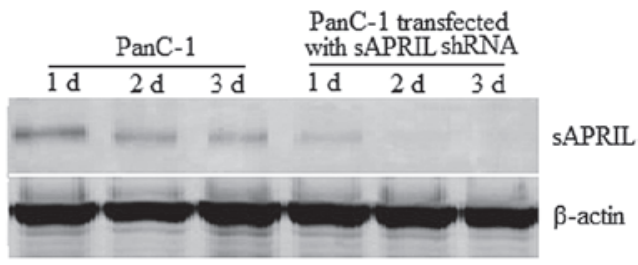

D

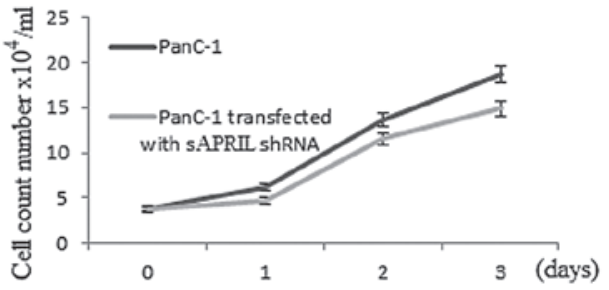

Figure 4. sAPRIL levels in non-transfected and sAPRIL shRNA-transfected PanC-1 cells, and the decrease in growth rate of transfected cells. (A) Reverse transcription-quantitative PCR analysis reveals lower levels of the sAPRIL mRNA in transfected compared to non-transfected PanC-1 cells. (B) Western blot analysis showing lower levels of the sAPRIL protein in transfected compared to non-transfected PanC-1 cells. (C) Western blot analysis of the levels of sAPRIL in the serum of non-transfected and transfected PanC-1 cells. (D) sAPRIL silencing inhibits the growth rate of shRNA-transfected PanC-1 cells compared to non-transfected cells. Each bar represents the mean \pm SD of three independent experiments. sAPRIL, soluble a-proliferation-inducing ligand; shRNA, short hairpin RNA.

recurrence or metastasis, was observed in only $10 \%$ of the subjects. According to the TNM classification system (25), six different tissue samples were obtained from the five-year survivors and marked as normal (healthy) pancreatic tissues, TNM stage I, IIA, IIB, III and IV. The protein levels of sAPRIL gradually and significantly $(\mathrm{P}<0.01)$ increased from normal pancreatic tissues to PA tissues at TNM stage IV (Table I). Immunohistochemical analysis confirmed that the protein level of sAPRIL increased from the normal pancreatic (Fig. 1A) to the PA tissues (Fig. 1B-F). sAPRIL was highly expressed at the advanced stages of PA (Fig. 1E and F). The factor analysis indicated that the serum sAPRIL level is a suitable prognostic marker for the development of PA. According to the univariate analysis results, tumor size ( $>3$ vs. $<3 \mathrm{~cm}$ ), the presence of lymph node metastasis, the TNM classification stage (I, IIA, IIB, III and IV) and the type of tissues significantly correlated to the level of sAPRIL (Table I).

The serum SAPRIL level is associated with the development of $P A$. Pancreatic biopsies of five-year survivors were collected by cystoscopy and stored at $-80^{\circ} \mathrm{C}$. A total of 160 tissue samples ( $\mathrm{n}=80$ for normal pancreatic tissues, $\mathrm{n}=18$ for TNM stage I, $\mathrm{n}=10$ for TNM stage IIA, $\mathrm{n}=8$ for TNM stage IIB, $\mathrm{n}=20$ for TNM stage III, and $n=24$ for TNM stage IV) were examined by a pathologist experienced with PA at the Departments of Hepatobiliary and Pancreas Surgery and Pathology, The General Hospital of Shenyang Military Region (Shenyang, China). To examine the clinical relevance of the sAPRIL level, we investigated whether the sAPRIL mRNA and protein levels are associated with the PA stage of the patients. From TNM stage I to IV, the mRNA levels of $S A P R I L$ gradually increased 
in the PA tissues (Fig. 2A). The mRNA level of $s A P R I L$ remained low in the adjacent healthy pancreatic tissues (Fig. 2A). Similar to the mRNA, the protein levels of sAPRIL increased with the development of PA (Fig. 2B). The sAPRIL protein was not detected in the adjacent healthy pancreatic tissues (Fig. 2B). To explore a non-invasive method for the diagnosis of PA, we also examined the serum sAPRIL levels at different stages of the disease. As expected, the serum sAPRIL levels gradually increased from stages I to IV (Fig. 2C).

The growth rate of $P A$ cells is increased upon transfection with the SAPRIL expression vector. We enquired whether sAPRIL is expressed at the mRNA and protein level in PA cell lines transfected with the sAPRIL expression vector using RT-qPCR (Fig. 3A) and western blot analysis (Fig. 3B). Furthermore, we investigated the possibility that SAPRIL is secreted from PA cells. Western blot analysis of the secreted protein fraction showed that the sAPRIL protein is present in the medium and is increased when the PA cells were transfected with the SAPRIL expression vector (Fig. 3C). These results indicated that the PA PanC-1 cells may secrete sAPRIL. Compared to the PA non-transfected PanC-1 cells, the growth rate of the transfected cells increased up to $27 \%(\mathrm{P}<0.01)$ after a three-day culture (Fig. 3D).

The growth rate of PA cells is decreased when cells are transfected with SAPRIL shRNA. The enhanced growth rate of the cells with an increased serum level of sAPRIL suggests that this protein may be involved in cancer progression. We therefore examined whether gene silencing of $S A P R I L$ affects the growth rate of PA PanC-1 cells. RT-qPCR and western blot analysis showed that sAPRIL is present in the PanC-1 cells that were not transfected and absent in the cell lines transfected with the $S A P R I L$ shRNA (Fig. 4A and B). Similarly, the protein SAPRIL was present in the medium of the cultured non-transfected PanC-1 cells and absent in the medium of the cells transfected with sAPRIL shRNA (Fig. 4C). Compared to the non-transfected cells, the growth rate of the cells decreased up to $19 \%(\mathrm{P}<0.01)$ when they were transfected with the sAPRIL shRNA after a three-day culture (Fig. 4D).

\section{Discussion}

Detection of PA, particularly at early stages, remains a great challenge. Recently, serum biomarkers have drawn increasing attention in the search for less invasive tools for the diagnosis of numerous types of cancer (27-32). Serum biomarkers for the diagnosis of PA have been also widely reported (33-37). For instance, CYFRA 21-1 levels were found to be significantly associated with the PA stage and may serve as a valuable tool for the treatment and prognosis of advanced PA (37). The aberrant expression of microRNAs (miRNAs) in various tissues is also related to various types of cancer. miRNAs are present in the serum and the plasma of humans. Furthermore, the levels of miRNAs in the serum are stable, reproducible, and consistent in the same individuals (38). Thus, serum miRNA may also be suitable as a biomarker for the diagnosis of cancer. In a recent study, seven miRNAs showed different levels of expression in PA compared to those observed in the control group. The 7 miRNA-based biomarker assay showed high sensitivity to distinguish the different stages of PA, and was thus proposed as a novel non-invasive diagnostic tool (33). Although numerous serum biomarkers have been reported, none has been applied in the clinic to date. Thus, it is still necessary to find novel biomarkers for the early diagnosis of PA.

Surgery is the primary option for the treatment of cancer, but the survival rate is still low due to recurrence and metastasis (39). Thus, early diagnosis is of high importance. EMT plays a critical role in cell invasion, migration and metastasis (40), which is closely related to the recurrence and metastasis of cancer after surgery. sAPRIL is a member of the TNF family $(41,42)$ and may be the inducer of MET (43-46). This prompted us to explore the functions of sAPRIL in PA in this study. sAPRIL was found to be highly expressed in PA tissues, but expressed at low levels in healthy pancreatic tissues adjacent to the cancerous cells. These findings were supported by the mRNA and protein level detection of sARPIL by RT-qPCR and western blot analysis, respectively. We also found that the serum SAPRIL level is higher in PA patients compared to the controls.

The high growth rate of cells transfected with the sARPIL expression vector, along with the high expression of sAPRIL in PA cells, suggest that SARPIL promotes the development of PA (Fig. 3D). To confirm this finding, sAPRIL shRNA was used. The results showed that the inhibition of SAPRIL expression inhibits growth of the PA cell line PanC-1 (Fig. 4D). Therefore, sAPRIL can be considered as a novel enhancer of PA progression. The elevated level of serum sAPRIL offers a convenient, less invasive tool for the early diagnosis of PA. To further understand the molecular mechanism underlying the increased serum expression of sAPRIL, the relationship between sAPRIL and EMT will be investigated in future work.

In conclusion, we demonstrated that serum levels of sAPRIL, which appeared to be secreted by PA cells, show good predictive value for the prognosis of PA. Further research to explore whether sAPRIL promotes the EMT in PA cells after long-term surgery is however urgently needed. Overall, sAPRIL is a promising target to develop for therapeutic treatment of PA.

\section{References}

1. Zhang R, Humphreys I, Sahu RP, Shi Y and Srivastava SK: In vitro and in vivo induction of apoptosis by capsaicin in pancreatic cancer cells is mediated through ROS generation and mitochondrial death pathway. Apoptosis 13: 1465-1478, 2008.

2. Stathis A and Moore MJ: Advanced pancreatic carcinoma: current treatment and future challenges. Nat Rev Clin Oncol 7: 163-172, 2010.

3. Aparicio JR, Martínez J, Niveiro M, et al: Direct intracystic biopsy and pancreatic cystoscopy through a 19-gauge needle EUS (with videos). Gastrointest Endosc 72: 1285-1288, 2010.

4. Falco A, Rosati A, Festa M, et al: BAG3 is a novel serum biomarker for pancreatic adenocarcinomas. Am J Gastroenterol 108: 1178-1180, 2013.

5. Kosanam H, Prassas I, Chrystoja CC, et al: Laminin, gamma 2 (LAMC2): a promising new putative pancreatic cancer biomarker identified by proteomic analysis of pancreatic adenocarcinoma tissues. Mol Cell Proteomics 12: 2820-2832, 2013.

6. Wang Y, Kuramitsu Y, Ueno T, et al: Proteomic differential display identifies upregulated vinculin as a possible biomarker of pancreatic cancer. Oncol Rep 28: 1845-1850, 2012.

7. Wang T, Wentz SC, Ausborn NL, et al: Pattern of breast cancer susceptibility gene 1 expression is a potential prognostic biomarker in resectable pancreatic ductal adenocarcinoma. Pancreas 42: 977-982, 2013.

8. Tempero MA, Klimstra D, Berlin J, et al: Changing the way we do business: recommendations to accelerate biomarker development in pancreatic cancer. Clin Cancer Res 19: 538-540, 2013. 
9. Ray P, Sullenger BA and White RR: Further characterization of the target of a potential aptamer biomarker for pancreatic cancer: cyclophilin B and its posttranslational modifications. Nucleic Acid Ther 23: 435-442, 2013

10. Ray P, Rialon-Guevara KL, Veras E, Sullenger BA and White RR: Comparing human pancreatic cell secretomes by in vitro aptamer selection identifies cyclophilin B as a candidate pancreatic cancer biomarker. J Clin Invest 122: 1734-1741, 2012.

11. Ono M, Kamita M, Murakoshi Y, et al: Biomarker discovery of pancreatic and gastrointestinal cancer by 2DICAL: 2-dimensional image-converted analysis of liquid chromatography and mass spectrometry. Int J Proteomics 2012: 897412, 2012.

12. Lyn-Cook BD, Yan-Sanders Y, Moore S, Taylor S, Word B and Hammons GJ: Increased levels of NAD $(\mathrm{P}) \mathrm{H}$ : quinone oxidoreductase 1 (NQO1) in pancreatic tissues from smokers and pancreatic adenocarcinomas: A potential biomarker of early damage in the pancreas. Cell Biol Toxicol 22: 73-80, 2006.

13. Lau C, Kim Y, Chia D, et al: Role of pancreatic cancer-derived exosomes in salivary biomarker development. J Biol Chem 288: 26888-26897, 2013.

14. Hahne M, Kataoka T, Schröter M, et al: APRIL, a new ligand of the tumor necrosis factor family, stimulates tumor cell growth J Exp Med 188: 1185-1190, 1998.

15. Chuang, MJ, Sun KH, Tang SJ, Deng MW, Wu YH, Sung JS Cha TL and Sun GH: Tumor-derived tumor necrosis factor-alpha promotes progression and epithelial-mesenchymal transition in renal cell carcinoma cells. Cancer Sci 99: 905-913, 2008.

16. Takahashi E, Nagano O, Ishimoto T, Yae T, Suzuki Y, Shinoda T, Nakamura S, Niwa S, Ikeda S and Koga H: Tumor necrosis factor- $\alpha$ regulates transforming growth factor- $\beta$-dependent epithelial-mesenchymal transition by promoting hyaluronan-CD44-moesin interaction. J Biol Chem 285: 4060-4073, 2010.

17. Dillon SR, Harder B, Lewis KB, et al: B-lymphocyte stimulator/a proliferation-inducing ligand heterotrimers are elevated in the sera of patients with autoimmune disease and are neutralized by atacicept and B-cell maturation antigen-immunoglobulin. Arthritis Res Ther 12: R48, 2010.

18. Xin G, Cui Z, Su Y, Xu LX, Zhao MH and Li KS: Serum BAFF and APRIL might be associated with disease activity and kidney damage in patients with anti-glomerular basement membrane disease. Nephrology (Carlton) 18: 209-214, 2013.

19. Pollard RP, Abdulahad WH, Vissink A, et al: Serum levels of BAFF, but not APRIL, are increased after rituximab treatment in patients with primary Sjogren's syndrome: data from a placebo-controlled clinical trial. Ann Rheum Dis 72: 146-148, 2013.

20. Gheita TA, Raafat H, Khalil H and Hussein H: Serum level of APRIL/BLyS in Behcet's disease patients: clinical significance in uveitis and disease activity. Mod Rheumatol 23: 542-546, 2013.

21. Kiyama K, Kawabata D, Hosono Y, et al: Serum BAFF and APRIL levels in patients with IgG4-related disease and their clinical significance. Arthritis Res Ther 14: R86, 2012.

22. Wang F, Chen L, Ding W, et al: Serum APRIL, a potential tumor marker in pancreatic cancer. Clin Chem Lab Med 49: 1715-1719, 2011.

23. Tecchio C, Nichele I, Mosna F, et al: A proliferation-inducing ligand (APRIL) serum levels predict time to first treatment in patients affected by B-cell chronic lymphocytic leukemia. Eur J Haematol 87: 228-234, 2011.

24. Ding W, Wang J, Wang F, et al: Serum sAPRIL: a potential tumor-associated biomarker to colorectal cancer. Clin Biochem 46: 1590-1594, 2013

25. Sobin LH, Gospodarowicz MK and Wittekind C (eds): TNM Classification of Malignant Tumours. Vol 28. 7th edition. Wiley-Blackwell, West Sussex, UK, pp231-234, 2009.

26. Takano S, Yoshitomi H, Togawa A, et al: Apolipoprotein C-1 maintains cell survival by preventing from apoptosis in pancreatic cancer cells. Oncogene 27: 2810-2822, 2008.
27. Blumenstein B, Saad F, Hotte S, et al: Reduction in serum clusterin is a potential therapeutic biomarker in patients with castration-resistant prostate cancer treated with custirsen. Cancer Med 2: 468-477, 2013.

28. Wu Y, Du X, Xue C, et al: Quantification of serum SOX2 DNA with FQ-PCR potentially provides a diagnostic biomarker for lung cancer. Med Oncol 30: 737, 2013.

29. Harima Y, Ikeda K, Utsunomiya K, et al: Apolipoprotein C-II is a potential serum biomarker as a prognostic factor of locally advanced cervical cancer after chemoradiation therapy. Int J Radiat Oncol Biol Phys 87: 1155-1161, 2013.

30. Toiyama Y, Hur K, Tanaka K, et al: Serum miR-200c is a novel prognostic and metastasis-predictive biomarker in patients with colorectal cancer. Ann Surg 259: 735-743, 2014.

31. Wang J, Wang X, Lin S, et al: Identification of kininogen-1 as a serum biomarker for the early detection of advanced colorectal adenoma and colorectal cancer. PLoS One 8: e70519, 2013.

32. Biskup K, Braicu EI, Sehouli J, et al: Serum glycome profiling: a biomarker for diagnosis of ovarian cancer. J Proteome Res 12: 4056-4063, 2013.

33. Liu R, Chen X, Du Y, et al: Serum microRNA expression profile as a biomarker in the diagnosis and prognosis of pancreatic cancer. Clin Chem 58: 610-618,2012.

34. Eguchi H, Ishikawa O, Ohigashi $\mathrm{H}$, et al: Serum REG4 level is a predictive biomarker for the response to preoperative chemoradiotherapy in patients with pancreatic cancer. Pancreas 38 791-798, 2009.

35. Dutta SK, Girotra M, Singla M, et al: Serum HSP70: a novel biomarker for early detection of pancreatic cancer. Pancreas 41: 530-534, 2012

36. Brand RE, Nolen BM, Zeh HJ, et al: Serum biomarker panels for the detection of pancreatic cancer. Clin Cancer Res 17: 805-816, 2011.

37. Boeck S, Wittwer C, Heinemann V, et al: Cytokeratin 19-fragments (CYFRA 21-1) as a novel serum biomarker for response and survival in patients with advanced pancreatic cancer. Br J Cancer 108: 1684-1694, 2013.

38. Chen X, Ba Y, Ma L, et al: Characterization of microRNAs in serum: a novel class of biomarkers for diagnosis of cancer and other diseases. Cell Res 18: 997-1006, 2008.

39. Sugiura T, Uesaka K, Mihara K, et al: Margin status, recurrence pattern, and prognosis after resection of pancreatic cancer. Surgery 154: 1078-1086, 2013.

40. Kitamura K, Seike M, Okano T, et al: MiR-134/487b/655 cluster regulates TGF- $\beta$-induced epithelial-mesenchymal transition and drug resistance to gefitinib by targeting MAGI2 in lung adenocarcinoma cells. Mol Cancer Ther 13: 444-453, 2014.

41. Mackay F and Ambrose C: The TNF family members BAFF and APRIL: the growing complexity. Cytokine Growth Factor Rev 14: 311-324, 2003.

42. Lascano V, Zabalegui LF, Cameron K, et al: The TNF family member APR IL promotes colorectal tumorigenesis. Cell Death Differ 19: 1826-1835, 2012.

43. Watanabe T, Takahashi A, Suzuki K, et al Epithelial-mesenchymal transition in human gastric cancer cell lines induced by TNF- $\alpha$-inducing protein of Helicobacter pylori. Int J Cancer 134: 2373-2382, 2014.

44. Wang H, Fang R, Wang XF, et al: Stabilization of Snail through AKT/GSK-3 $\beta$ signaling pathway is required for $\mathrm{TNF}-\alpha$-induced epithelial-mesenchymal transition in prostate cancer PC3 cells. Eur J Pharmacol 714: 48-55, 2013.

45. Wang $\mathrm{H}$, Wang HS, Zhou BH, et al: Epithelial-mesenchymal transition (EMT) induced by TNF- $\alpha$ requires AKT/GSK-3 $\beta$-mediated stabilization of snail in colorectal cancer. PLoS One 8: e56664, 2013.

46. Techasen A, Namwat N, Loilome W, et al: Tumor necrosis factor- $\alpha$ (TNF- $\alpha$ ) stimulates the epithelial-mesenchymal transition regulator Snail in cholangiocarcinoma. Med Oncol 29: 3083-3091, 2012. 